\title{
Repeated Dexamethasone Intravitreal Implant for the Treatment of Diabetic Macular Oedema Unresponsive to Anti-VEGF Therapy: Outcome and Predictive SD-OCT Features
}

\author{
Katja Hatz ${ }^{\mathrm{a}, \mathrm{b}}$ Andreas Ebneter ${ }^{\mathrm{c}}$ Cengiz Tuerksever ${ }^{\mathrm{a}}$ Christian Pruente ${ }^{\mathrm{b}, \mathrm{d}}$ \\ Martin Zinkernage ${ }^{c}$ \\ ${ }^{a}$ Vista Klinik, Binningen, Switzerland; ${ }^{b}$ Department of Ophthalmology, University of Basel, Basel, Switzerland; \\ 'Department of Ophthalmology, Inselspital, Bern, Switzerland; ${ }^{d}$ Department of Ophthalmology, Kantonsspital \\ Liestal, Liestal, Switzerland
}

\section{Keywords}

Dexamethasone implant $\cdot$ Diabetic macular oedema .

Optical coherence tomography · Photoreceptor integrity

\begin{abstract}
Purpose: To investigate dexamethasone intravitreal implant $0.7 \mathrm{mg}$ (DEX implant) for the treatment of diabetic macular oedema (DME) refractory to anti-vascular endothelial growth factor (anti-VEGF) therapy and evaluate predictive factors. Methods: Two-centre retrospective interventional case series, including 40 eyes of 31 patients treated with DEX implant for at least 2 consecutive cycles. Results: Mean \pm SD intervals from implantation to recurrence in the first $(4.2 \pm$ 1.0 months) and second cycles ( $4.0 \pm 0.9$ months) were not significantly different. Best corrected visual acuity improved significantly $(p<0.001)$ by $7.0 \pm 8.4$ letters from baseline to month 2 , and by $5.1 \pm 6.9$ letters between the first and second cycles. Central retinal thickness reduction 2 months after implantation was greater after the first $(-194 \pm 172 \mu \mathrm{m})$ than the second cycle $(-134 \pm 150 \mu \mathrm{m})$. Ellipsoid zone-external limiting membrane (EZ-ELM) disruption score decreased from $1.39 \pm 1.16$ at baseline to $1.24 \pm 1.16(p=0.0832)$ after cycle 1 and remained stable 2 months after cycle 2 . Eyes with
\end{abstract}

\section{KARGER}

(c) 2018 S. Karger AG, Basel

E-Mail karger@karger.com

www.karger.com/oph persisting severe EZ-ELM disruption (score $>2, n=10$ ) 2 months after the first DEX implant showed significantly $(p=$ $0.0153)$ smaller visual acuity (VA) gains than eyes with less severe (score $\leq 2$ ) EZ-ELM disruption. Conclusion: Repeated intravitreal DEX injections with average intervals of 4 months are valuable in patients with DME refractory to anti-VEGF therapy. Disorganization of outer retinal layers (EZ-ELM) may predict smaller VA gains if evaluated after initial reduction of macular oedema.

(c) 2018 S. Karger AG, Basel

\section{Introduction}

Diabetic macular oedema (DME) is one of the leading causes of vision loss and blindness in patients with diabetes [1]. Laser photocoagulation was the standard of care for the treatment of DME until the availability of antivascular endothelial growth factor (anti-VEGF) therapies. Studies have demonstrated improved visual and anatomical outcomes with anti-VEGF therapies, with welltolerated safety profiles [2-6]. However, a retrospective analysis of the RIDE/RISE studies showed that after 36 months of monthly treatment with ranibizumab, $18 \%$ of 
patients ( $n=88 / 500)$ still needed further high-frequency treatment defined as $>7$ injections per year [7], indicating that some patients may be refractory to anti-VEGF treatment.

DME features characteristics of chronic, local inflammatory response in patients who do not respond to antiVEGF therapy or need very frequent treatment; therefore, inflammatory mediators other than VEGF may also play a role in sustaining DME [8]. Vitreous fluid from patients with DME shows evidence of active inflammation, containing elevated concentrations of many different inflammatory and pro-inflammatory mediators [911]. Therefore, treatment targeting these inflammatory or pro-inflammatory mediators may improve disease outcomes.

Intravitreal triamcinolone acetonide, a corticosteroid, has been shown to be effective in treating anti-VEGF-resistant DME, but only in the short term [12]. The dexamethasone intravitreal implant $0.7 \mathrm{mg}$ (DEX implant, Ozurdex $^{\circledR}$, Allergan Inc., Irvine, CA, USA), a long-acting sustained-release corticosteroid [13], has been shown to be effective in treating DME [14-17]. Upon treatment with DEX implant, pro-permeability factors were reduced in aqueous samples [18].

Most studies and case series have reported outcomes either following a single DEX implant $[14,17,19,20]$ or retreatment with DEX implant after 6 months $[15,16]$. One small retrospective analysis reported the use of repeated as-needed DEX implants [21].

Until now, there have been limited data regarding predictive morphological features in DME that can identify patients who may benefit from DEX implant treatment. Coscas et al. [22] reported a case series of 9 patients with macular oedema due to retinal vein occlusion and showed that visual acuity (VA) in eyes with interrupted or thickened photoreceptor inner and outer segment junctions (ellipsoid zone, EZ) at 3 months was worse than in eyes with a normal EZ. Bansal et al. [23] suggested that the preservation of external limiting membrane (ELM) and EZ in eyes with uveitic cystoid macular oedema might carry a better visual prognosis after DEX implantation, whereas retinal thickness, height of cysts, and presence of serous retinal detachment did not affect the final visual outcome. So far, there are no reports that spectral domain optical coherence tomography (SD-OCT) scans of patients with DME and a DEX implant show any features that would predict the outcome of the treatment, but it seems likely that there might be a correlation $[24,25]$.

This multicentre retrospective case series study investigated the effect of as-needed treatment with repeated
DEX implants in patients with DME not responsive to anti-VEGF therapy and evaluated predictive morphological features.

\section{Materials and Methods}

\section{Study Design}

This was a multicentre (2 centres), retrospective, consecutive case series performed at Vista Klinik, Binningen, and Inselspital, Bern, Switzerland, to investigate the use of the DEX implant in routine clinical use for the treatment of DME in patients not responding to anti-VEGF therapy, and to identify features using SDOCT that may predict treatment outcome.

The study was carried out in line with the Declaration of Helsinki, and with guidelines of the International Conference on Harmonisation, Good Clinical Practice, and the Humanforschungsgesetz (law on research on human beings in Switzerland). Ethics approval number: LEK EKNZ 2014-360, EK BE 308/14.

Written informed consent was obtained from each participating patient for retrospective analyses of patient data and use of imaging material obtained from the participating centres.

\section{Patients}

The study retrospectively evaluated all eyes with chronic DME (confirmed with fluorescein angiography) treated with the DEX implant for at least 2 consecutive cycles (i.e., until recurrence of symptoms after second implant) from 2011 to 2015 . All patients had been pre-treated with routine anti-VEGF therapy and required either very frequent treatment (monthly) or failed to show morphological improvement under anti-VEGF therapy. Lesions that had not been fully documented were excluded.

\section{Outcomes and Assessments}

Mean treatment interval from implantation to recurrence, defined as recurrence of sub- or intra-retinal fluid in OCT, after the first or second injection with DEX was the primary endpoint of the study. Secondary outcome measures included mean change in best corrected visual acuity (BCVA) and central retinal thickness (CRT) from baseline to month 2 , as well as time of recurrence after each injection, and comparison between the two consecutive injections with DEX; mean EZ-ELM disruption score at baseline and 2 months after first and second implant and its correlation with VA outcome; mean number of hyperreflective foci (HRF) at baseline and 2 months after first and second implant and its correlation with VA outcomes; and safety, including cataract progression, mean change in intraocular pressure (IOP) from baseline to month 2, and time of recurrence for each cycle and between cycles. BCVA was either measured as 4-month VA following ETDRS refraction protocol or as Snellen 4- or 6-month BCVA and transferred into ETDRS letter score. IOP was measured using Goldmann applanation tonometry.

EZ-ELM disruption, as measured by SD-OCT (Heidelberg Engineering, Inc., Heidelberg, Germany), was evaluated as the mean of the horizontal and vertical scans and graded as: 0 (no disruption in 1 -mm centre), 1 (mild disruption $<1 / 4$ within 1 -mm centre), 2 ( $1 / 4$ to $3 / 4$ disruption within $1-m m$ centre), and 3 (>3/4 disruption within $1-\mathrm{mm}$ centre). HRF number (central $1 \mathrm{~mm}$ ) was calculated as the sum of the HRF numbers within the horizontal and vertical scans using SD-OCT. 
Table 1. Baseline characteristics $(n=40)$

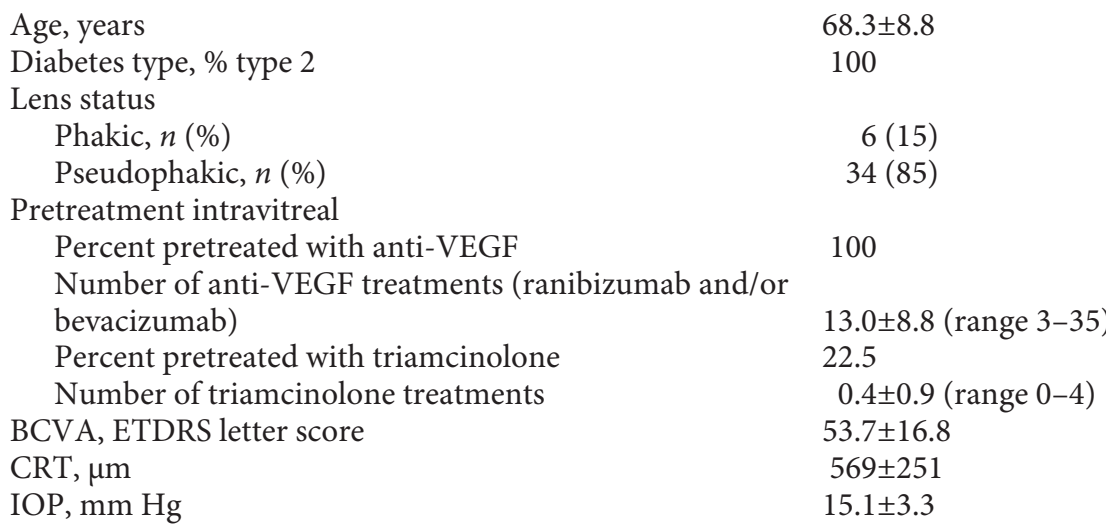

Unless otherwise stated, results are expressed as mean \pm SD. BCVA, best corrected visual acuity; CRT, central retinal thickness; EDTRS, early treatment for diabetic retinopathy study; IOP, intraocular pressure; VEGF, vascular endothelial growth factor.
SD-OCT scans were acquired using established protocols compromising 19 or 49 6-mm horizontal scans (volume scan) in a follow-up modus (high speed modus, 9 frames, 512 A-scans) as well as the 6-mm star scan (high speed modus, 9 frames, 512 A-scans). For thickness analyses, a manual correction of the automated segmentations was performed if any segmentation errors were present. For analyses which related to the central vertical and horizontal scan, the 6-mm star scan was used.

\section{Statistical Analysis}

For the variables, mean \pm standard deviation (SD) or percentages were calculated. Statistical analyses were performed with SPSS version 23.0 for Windows (SPSS; Chicago, IL, USA). To compare follow-up with baseline values, a 2 -sided $t$ test was performed. The comparison of before and after treatment values in the same group was performed with the $t$ test for dependent variables, while intergroup comparisons were performed with the $t$ test for independent variables. A $p$ value $<0.05$ was considered statistically significant.

Statistical analyses were only performed with data from the first two treatment cycles due to the decreasing number of subjects from cycle 3 onwards.

\section{Results}

\section{Patient Characteristics and Demographics}

Overall, 40 DME lesions from 31 patients were evaluated in this analysis (23 in Binningen; 17 in Bern). One further patient was not included in this analysis owing to loss of follow-up during the second cycle. The mean age of patients was $68.3 \pm 8.8$ years and the mean BCVA and CRT at baseline were $53.7 \pm 16.8$ letters and $569 \pm 251 \mu \mathrm{m}$, respectively. Patient baseline characteristics are provided in Table 1.

DEX Implant in DME and SD-OCT

Predictive Factors

\section{Mean Treatment Interval for Each Cycle}

Overall, the eyes received a mean of $3.72 \pm 2.41 \mathrm{DEX}$ implants (range 2-13) during the study period. The mean interval from implantation to recurrence of DME in the first cycle (4.2 \pm 1.0 months) and the second cycle ( $4.0 \pm$ 0.9 months) was not significantly different $(p=0.419)$. In further cycles, mean interval from implantation to recurrence of DME was similar to that observed in the first 2 cycles $(4.3 \pm 1.2$ and $4.1 \pm 0.5$ months in the third and fourth cycles, respectively).

\section{Change in BCVA between First and Second Cycles}

Mean VA improved significantly $(p<0.001)$ by $7.0 \pm$ 8.4 letters from baseline to month 2 and by $5.1 \pm 6.9$ letters within the first and second cycle. Mean VA observed at the baseline of the first cycle (BL1, $53.7 \pm 16.8$ letters) was not significantly different from that observed at the baseline of the second cycle (BL2; $54.2 \pm 16.9$ letters, $p=$ 0.729) (Fig. 1a). Similarly, mean VA observed 2 months after implantation in the first and second cycles $(60.7 \pm$ 16.4 and $59.3 \pm 17.0$ letters, $p=0.164)$, and during recurrence between the first and second cycles (54.8 \pm 16.6 and $52.9 \pm 17.3$ letters, respectively; $p=0.193$ ) was not significantly different.

Two months after implantation, eyes with lower baseline VA ( $\leq 39$ letters) tended to show a greater VA gain than eyes with higher baseline VA ( $\geq 60$ letters) in the first cycle ( $p=0.073$, see Table 2$)$; however, no such differences were noted in the second cycle.

VA outcomes for pseudophakic and phakic eyes are shown in Figure 1b, c. In phakic eyes, VA was higher at 


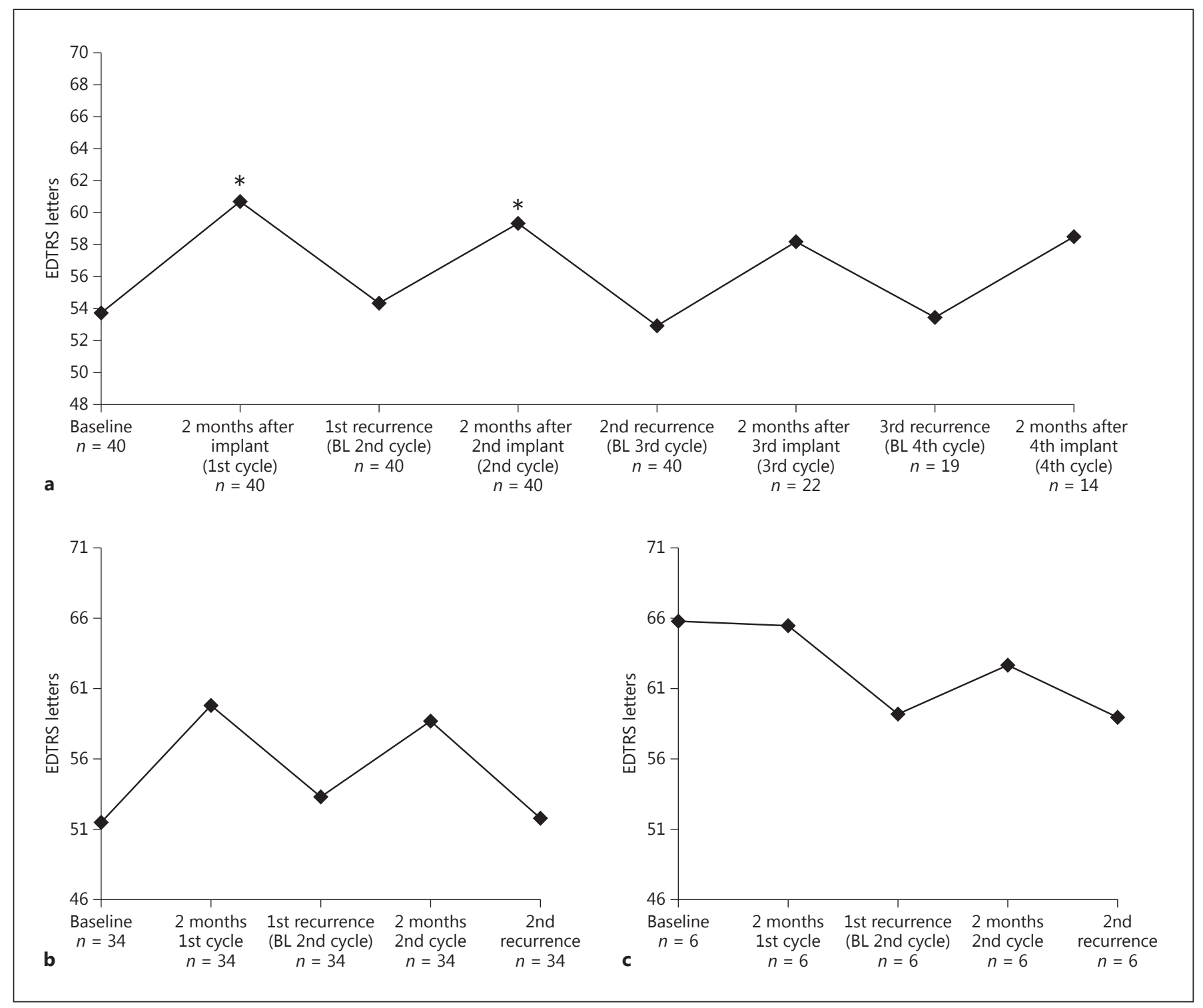

Fig. 1. Change in visual acuity from baseline to 2 months after each treatment and at recurrence for all eyes (a); pseudophakic eyes (b); and phakic eyes (c). BL, baseline. ${ }^{*} p<0.001$ from baseline to post-implant.

baseline ( $\geq 60$ letters); but it then dropped to $<60$ letters 2 months after DEX implant during the first and second cycle (Fig. 1b, c).

\section{Change in CRT: Comparison between First and \\ Second Cycles}

DEX significantly reduced mean CRT from baseline to month 2 within each of the two cycles (1st cycle: from 569 \pm 251 to $375 \pm 199 \mu \mathrm{m}, p<0.001 ; 2$ nd cycle: from $513 \pm$ 241 to $379 \pm 183 \mu \mathrm{m}, p<0.001)$. Mean CRT at baseline for the first cycle $(569 \pm 251 \mu \mathrm{m})$ was significantly greater than mean CRT at baseline for the second cycle (513 \pm $241 \mu \mathrm{m} ; p=0.047$ ) (Fig. 2). Mean CRT reduction observed 2 months after DEX implantation in the first cycle $(-194 \pm 172 \mu \mathrm{m})$ was significantly greater than that observed in the second cycle $(-134 \pm 150 \mu \mathrm{m}, p=0.025)$. However, there was no significant difference in mean CRT values 2 months after the first $(375 \pm 199 \mu \mathrm{m})$ and second $(379 \pm 183 \mu \mathrm{m})$ implants $(p=0.833)$, or after recurrence in the 2 cycles (first: $498 \pm 238 \mu \mathrm{m}$ and second: 


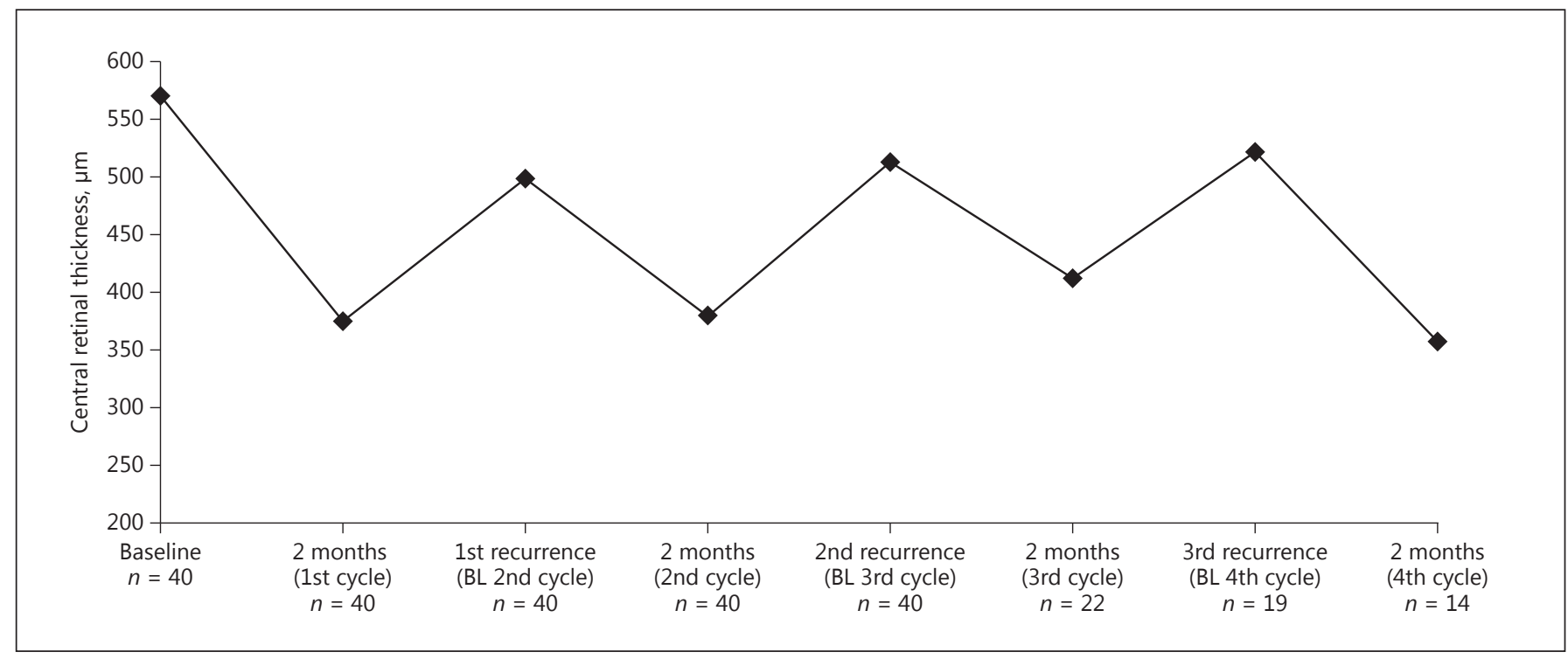

Fig. 2. Change in central retinal thickness of all eyes 2 months after each treatment and at recurrence. BL, baseline.

Table 2. Visual acuity gain in 2 implant cycles and assessment of correlation with baseline parameters in patients with DME ( $t$ test for independent variables)

\begin{tabular}{|c|c|c|c|c|}
\hline \multirow[t]{2}{*}{ Parameter } & \multicolumn{2}{|c|}{ VA gain 2 months after 1 st implant } & \multicolumn{2}{|c|}{$\begin{array}{l}\text { VA gain } 2 \text { months after } 2 \text { nd implant } \\
\text { (calculated from baseline of } 2 \text { nd cycle) }\end{array}$} \\
\hline & mean \pm SD & $p$ value & mean \pm SD & $p$ value \\
\hline \multicolumn{5}{|l|}{ Baseline BCVA } \\
\hline$\leq 39$ letters & $12.5 \pm 10.3(n=11)$ & \multirow[t]{2}{*}{0.073} & $6.6 \pm 7.9(n=11)$ & \multirow[t]{2}{*}{0.3533} \\
\hline$\geq 60$ letters & $6.9 \pm 5.9(n=18)$ & & $4.4 \pm 4.6(n=18)$ & \\
\hline \multicolumn{5}{|l|}{ Baseline CRT } \\
\hline$\leq 400 \mu \mathrm{m}$ & $3.9 \pm 3.8(n=10)$ & \multirow[t]{2}{*}{0.0343} & $2.2 \pm 2.6(n=10)$ & \multirow[t]{2}{*}{0.0173} \\
\hline$\geq 600 \mu \mathrm{m}$ & $11.1 \pm 9.2(n=10)$ & & $8.7 \pm 7.4(n=10)$ & \\
\hline \multicolumn{5}{|c|}{ Baseline EZ-ELM disruption } \\
\hline Score $>2$ & $5.8 \pm 6.3(n=11)$ & \multirow{2}{*}{0.5773} & $2.4 \pm 8.2(n=11)$ & \multirow{2}{*}{0.1257} \\
\hline Score $\leq 2$ & $7.5 \pm 9.6(n=29)$ & & $6.1 \pm 6.2(n=29)$ & \\
\hline \multicolumn{5}{|c|}{ EZ-ELM disruption after oedema reduction ( 2 months after 1 st implant) } \\
\hline Score $>2$ & $5.8 \pm 6.7(n=10)$ & \multirow[t]{2}{*}{0.6225} & $0.6 \pm 6.0(n=10)$ & \multirow[t]{2}{*}{0.0153} \\
\hline Score $\leq 2$ & $7.4 \pm 9.4(n=30)$ & & $6.6 \pm 6.6(n=30)$ & \\
\hline \multicolumn{5}{|c|}{ Hyperreflective foci } \\
\hline$>15$ & $3.1 \pm 12.0(n=8)$ & \multirow[t]{2}{*}{0.1638} & $5.5 \pm 11.0(n=8)$ & \multirow[t]{2}{*}{0.8478} \\
\hline$\leq 15$ & $8.0 \pm 7.7(n=32)$ & & $5.0 \pm 5.7(n=32)$ & \\
\hline
\end{tabular}

BCVA, best corrected visual acuity; CRT, central retinal thickness; DME, diabetic macular oedema; EZ-ELMELM, ellipsoid zone-external limiting membrane; SD, standard deviation. 
$513 \pm 223 \mu \mathrm{m}, p=0.445)$. Eyes with low baseline CRT $(\leq 400 \mu \mathrm{m})$ had significantly smaller VA gains than eyes with higher CRT $(\geq 600 \mu \mathrm{m})$ after the first and second cycle ( $p=0.0343$ and $p=0.0173$, respectively; Table 2 ).

\section{SD-OCT Morphological Findings and Their}

\section{Correlation to VA Outcome}

The mean EZ-ELM disruption score of $1.39 \pm 1.16$ at baseline decreased to $1.24 \pm 1.16$ at 2 months after the first DEX implant $(p=0.0832)$ and remained stable 2 months after the second DEX implant (1.24 \pm 1.10$)$. Severe EZELM disruption at baseline (score $>2$ ) did not seem to be predictive for VA gain 2 months after the first and the second implant: VA gains did not differ significantly between eyes with severe EZ-ELM disruption at baseline $(n=11$, score $>2)$ and those with no or less severe EZELM disruption at baseline (score $\leq 2 ; n=29$; Table 2). However, 2 months after the first DEX implant, eyes with persisting severe EZ-ELM disruption (score $>2, n=10$ ) showed significantly smaller $(p=0.0153)$ VA gains than did eyes with less severe (score $\leq 2$ ) EZ-ELM disruption $(0.6 \pm 6.0$ vs. $6.6 \pm 6.6$ letters, respectively, Table 2$)$.

The mean number of HRF within the central $1 \mathrm{~mm}$ per SD-OCT was $10.9 \pm 7.9$ at baseline, which decreased slightly to $9.1 \pm 7.62$ months after the first DEX implant $(p=0.0775)$ and remained stable after the second implant $(9.4 \pm 7.0, p=0.2285)$.

There was no significant difference in VA gains between eyes with high baseline HRF ( $>15 \mathrm{HRF}, n=8)$ and those with no or fewer HRF ( $\leq 15 \mathrm{HRF}, n=32)$ in cycle 1 $(p=0.1638)$ and cycle $2(p=0.8478)$ (Table 2$)$. High HRF at baseline seemed not to be predictive for VA gain 2 months after the first and second DEX implant. Similar results were seen after excluding the phakic eyes from analysis $(p=0.8962)$. In eyes with high $\operatorname{HRF}(>15, n=8)$ and those with no or less $\operatorname{HRF}(\leq 15, n=32)$, mean VA gains 2 months after first implant were found to be similar (4.6 \pm 7.6 and $5.2 \pm 6.7$ letters, $p=0.8044$; after exclusion of phakic eyes $p=0.8962$ ).

\section{Safety}

Intraocular Pressure

Baseline mean IOP of $15.1 \pm 3.3 \mathrm{~mm} \mathrm{Hg}$ increased significantly to $18.1 \pm 5.4 \mathrm{~mm} \mathrm{Hg} 2$ months after the first DEX implant $(p<0.001)$. However, there was no significant difference in IOP from baseline at the first recurrence $(15.4 \pm 4.1 \mathrm{~mm} \mathrm{Hg}, p=0.680)$. Overall, 8 eyes $(20 \%)$ were under topical IOP-lowering treatment at the end of the first cycle. For 2 eyes, the pre-existing topical IOPlowering treatment was continued; for 2 eyes, the pre- existing topical IOP-lowering treatment was expanded by adding another substance; and for 4 eyes, the topical IOPlowering treatment was newly established and continued after the treatment cycle finished.

IOP in the second cycle showed a similar trend to that of the first cycle, with mean IOPs of $15.2 \pm 3.7 \mathrm{~mm} \mathrm{Hg}$ at baseline, $18.4 \pm 7.9 \mathrm{~mm} \mathrm{Hg}$ at 2 months after implant, and $15.9 \pm 4.5 \mathrm{~mm} \mathrm{Hg}$ at recurrence. When compared with the baseline values, 2-month values and recurrence values demonstrated no significant differences between the first 2 cycles. Of 8 eyes in the first cycle, the topical IOP-lowering treatment was expanded for 3 eyes by adding another substance, 2 eyes received filtration surgery, and 3 were continued on their topical IOP-lowering treatment during the second cycle. Topical IOP-lowering treatment was newly established for 2 more eyes during the second cycle. During further cycles, topical treatment was stopped for 2 eyes, whereas it was newly established in 2 other eyes. There were no further changes in IOP management, and no eyes needed further filtration surgery.

\section{Cataract Progression}

At baseline, only 6 out of 40 (15\%) eyes were phakic. All these patients experienced cataract progression during follow-up and 1 eye received cataract surgery during the first cycle. Patients with phakic eyes (6/40) were younger (mean age: $58.8 \pm 7.7$ years) than the total population (see Table 1, $p=0.0173$ ), and these eyes showed significantly smaller VA changes 2 months after the first DEX implant compared with pseudophakic eyes $(-0.3$ \pm 11.3 vs. $8.3 \pm 7.7$ letters, $p=0.0238)$. One eye that received cataract surgery during the first cycle was included in the pseudophakic group for analysis of the second cycle. In the remaining 5 phakic eyes, a non-significant trend towards smaller gains in VA was observed compared with pseudophakic eyes $(1.2 \pm 9.2$ vs. $5.6 \pm 6.5$ letters, $p=0.1809)$. No other adverse events were observed.

\section{Discussion}

In this study, VA and CRT improved 2 months after DEX implant for 2 consecutive cycles with as-needed DEX treatment in eyes with DME refractory to antiVEGF therapy. No additional increase in cataract progression or IOP-related surgeries was observed. Patients with low VA and high CRT seemed to benefit the most from DEX treatment. Of the predictive factors assessed, disorganization of outer retinal layers (EZ-ELM disrup-
Hatz/Ebneter/Tuerksever/Pruente/ Zinkernagel 
tion) was predictive of lower VA gain after DEX implant in eyes with less severe oedema.

Alternative therapies are required for treatment of eyes with DME refractory to anti-VEGF treatment. Studies comparing the DEX implant to anti-VEGF treatment in DME showed similar gains in VA after 1 year of DEX treatment with reduced treatment rates (2.7/12 months) compared with patients receiving bevacizumab $(8.6 / 12$ months) [26]. In a prospective study, patients with refractory diffuse DME treated with DEX implant showed significant $(p<0.001)$ improvement in VA of 5-7 ETDRS letters until 6 months after implantation [27]. Similar significant improvement $(p=0.03)$ in VA 2 months after DEX implant (as monotherapy or combined with other treatments) was also reported by Gutierrez-Benitez et al. [28] in patients with DME refractory to ranibizumab. Overall, these studies reflect our findings that patients with DME refractory to anti-VEGF treatment can benefit from DEX treatment.

CRT was significantly reduced $(p<0.001)$ along with VA improvement after DEX implant in two multicentre, randomised studies over 3 years [15]. In our study, CRT decreased significantly 2 months after the first and second cycles. Although CRT was significantly greater at baseline 1 than at baseline 2 , this may be because re-treatment may have occurred a little earlier than primary DEX treatment due to the regular follow-up.

In clinical practice, the average number of DEX implants has been recorded at 2.3-2.5 per year [29]. Our results show that re-treatment with DEX after recurrence continued to improve VA and CRT. Improvements in VA after re-treatment have also been seen in other studies [30].

IOP needs to be carefully monitored after DEX implants but can be managed by topical IOP-lowering treatment in most patients. In our study, an increase in IOP was observed 2 months after treatment in both treatment cycles. Similar results were reported by Totan et al. [31] who showed a significant increase from baseline in IOP at 1 and 3 months $(p \leq 0.04)$ after implant; however, these were well controlled with topical treatment. Similar to our findings, Maturi et al. [30] reported a non-cumulative increase in IOP after sequential implants.

We found a non-significant trend of VA gains to be greater for eyes with low baseline VA ( $\leq 39$ letters) than for eyes with high baseline VA ( $\geq 60$ letters). Similarly, Bressler et al. [32] reported significantly $(p<0.001)$ larger gains in VA in eyes with low baseline VA scores compared with those in eyes with higher baseline VA scores. The lack of significance in our study may be due in part

DEX Implant in DME and SD-OCT

Predictive Factors to the low patient numbers. These data suggest that patients with low VA not responding to anti-VEGF may particularly benefit from DEX treatment.

In eyes with low baseline CRT $(\leq 400 \mu \mathrm{m})$, the VA gains within the first and second cycle were smaller than those in eyes with higher CRT $(\geq 600 \mu \mathrm{m})$. In 2013, the National Institute Health and Care Excellence (NICE) in the United Kingdom recommended the use of anti-VEGF treatment for DME, especially in the subgroup of patients with CRT $>400 \mu \mathrm{m}$ [33]. This recommendation was based on a clinical trial that demonstrated greater improvements in VA and CRT in eyes with CRT $>400 \mu \mathrm{m}$ at baseline than those with CRT of $<400 \mu \mathrm{m}$ [34]. Similar baseline parameters may also affect treatment outcomes with DEX in patients with chronic DME not responding to anti-VEGF treatment. Our results suggest that patients with high baseline CRT not responding to anti-VEGF may benefit from DEX treatment to a greater degree than those with lower CRT at baseline.

Standard treatment for DME involves repetitive, invasive intraocular injections, increasing the burden on patients, physicians and healthcare resources [35]. Biomarkers that could predict the effect of a therapy on future VA in eyes with DME could improve risk assessment, management decisions, and selection of eyes for clinical studies [35]. Disorganization of inner retinal layers as a predictor for VA outcome in DME within a cohort of mostly anti-VEGF- and/or laser-treated eyes was reported in a retrospective study by Sun et al. [35]. However, in our study, severe EZ-ELM disruption at baseline (score $>2$ ) was not associated with VA gain 2 months after the first or second DEX implant. Furthermore, no significant difference was noted between VA gains of eyes with severe EZ-ELM disruption at baseline (score $>2$ ) and those with no or less severe EZ-ELM disruption at baseline (score $\leq 2)$. In the patient population studied by Sun et al., mean baseline CRT $(408 \mu \mathrm{m})$ was much lower than that observed in our study $(569 \mu \mathrm{m})$, indicative of less severe macular oedema. The EZ-ELM disruption is more difficult to evaluate in eyes with more severe oedema, and this may explain the lack of correlation between baseline EZELM disruption score and VA outcome in our study. This may also explain our observation that after reduction of macular oedema at 2 months after the first implant, eyes with persisting severe EZ-ELM disruption (score $>2$ ) had a significantly smaller VA gain in the later follow-up. Therefore, in a population with severe macular oedema, evaluation of disorganization of outer retinal layers should be done after reduction of macular oedema as a predictor of later outcome. 
HRF have been reported in patients with DME [36]. Although the exact origin of these HRF remains unknown, reduction in HRF was seen after treatment with ranibizumab or DEX implant in patients with retinal vascular diseases, including retinal vein occlusions and DME [37]. In our study, we found a non-significant trend for HRF reduction 2 months after the first DEX implant ( $p=0.0775)$; the reduction remained stable for 2 months after the second implant. This lack of statistical significance may be due to the low patient numbers. Chatziralli et al. [37] further reported an association of the presence of HRF with poor VA in patients with macular oedema due to retinal vascular diseases. However, they did not observe better treatment success if the initial number of HRF was larger. In our study, high numbers of HRF at baseline ( $>15)$ also did not seem predictive for VA gain 2 months after the first and second DEX implant. However, our results cannot be directly compared with the study by Chatziralli's group because of the differences in total HRF calculations. In our study, total HRF numbers refer to the central $1 \mathrm{~mm}$ as a sum of the central horizontal and vertical scan (see Methods), whereas Chatziralli et al. [37] examined the horizontal 6-mm section with the most HRF present. Furthermore, our population consisted of a pre-treated population with chronic DME, which is indicated by the circumstance that all patients showed HRF within the central $1 \mathrm{~mm}$.

Some additional predictors that were not assessed in this study that may be of interest in the future are microaneurysms in the deep capillary plexus (DCP). Significant increases in the density of microaneurysms in DCP are observed in patients with DME and may contribute to the pathogenesis of DME [38]. Lee et al. [39] reported that DME eyes that responded poorly to anti-VEGF treatment tended to exhibit greater damage and more microaneurysms in the DCP. The study also reported that the degree of outer plexiform layer disruption in SDOCT corresponds well with the extent of DCP loss in DME eyes. Therefore, the extent of DCP loss and the corresponding outer plexiform layer disruption could be useful predictors of responsiveness to anti-VEGF treatment [39].

Unlike most published studies/case series that evaluated single implant or re-treatment after every 6 months, the present study evaluated the effect of treatment with repeated as-needed DEX implants for more than 2 treatment cycles. DEX implants are associated with risks including IOP elevation and cataract progression; therefore, they are often used as second-line therapy to
anti-VEGF in DME by many retina specialists [17]. For this reason, this study was evaluated in candidates for DEX implant who had chronic DME and needed frequent anti-VEGF treatment or who failed to respond to anti-VEGF treatment. This is also one of the first studies to investigate VA predictors, including SD-OCT parameters, in this population. Limitations of the study include the retrospective setting and small patient numbers.

In conclusion, this study shows that repeated intravitreal injections of DEX implants can successfully improve VA and reduce CRT in patients who are refractory to anti-VEGF therapy. The time for DEX re-injection for subsequent treatments should be tailored to each individual patient on a case-by-case basis to achieve optimum results. Patients with lower VA and/or greater CRT might benefit from DEX treatment. Disorganization of outer retinal layers (EZ-ELM) might predict smaller VA gains if evaluated after initial reduction of macular oedema. Further prospective studies are needed in this target population to confirm the predictors assessed in this study, to investigate other potentially predictive factors that may help identify patients who can benefit from early DEX treatment, and to compare outcomes in patients with DEX implants with outcomes in anti-VEGF non-responders.

\section{Acknowledgements}

Open Access fee was funded by Allergan. Editorial assistance (in the form of writing assistance, assembling tables and figures, collating author comments, grammatical editing, and referencing) was provided by Fishawack Indicia Ltd. UK and was funded by Allergan.

\section{Disclosure Statement}

Dr. Hatz has received honoraria for advisory board meetings and lectures and congress travel expenses from Novartis, Bayer, and Allergan.

Dr. Ebneter has received honoraria and travel support from Bayer, honoraria for advisory board meetings, lectures and congress travel support from Allergan, and non-financial support and a research grant from Novartis and Plexxikon Inc. 


\section{References}

1 Cheung N, Mitchell P, Wong TY: Diabetic retinopathy. Lancet 2010;376:124-136.

-2 Massin P, Bandello F, Garweg JG, Hansen LL, Harding SP, Larsen M, Mitchell P, Sharp D, Wolf-Schnurrbusch UE, Gekkieva M, Weichselberger A, Wolf S: Safety and efficacy of ranibizumab in diabetic macular edema (RESOLVE Study): a 12-month, randomized, controlled, double-masked, multicenter phase II study. Diabetes Care 2010;33:2399_ 2405.

-3 Nguyen QD, Brown DM, Marcus DM, Boyer DS, Patel S, Feiner L, Gibson A, Sy J, Rundle AC, Hopkins JJ, Rubio RG, Ehrlich JS; RISE and RIDE Research Group: Ranibizumab for diabetic macular edema: results from 2 phase III randomized trials: RISE and RIDE. Ophthalmology 2012;119:789-801.

4 Brown DM, Schmidt-Erfurth U, Do DV, Holz FG, Boyer DS, Midena E, Heier JS, Terasaki H, Kaiser PK, Marcus DM, Nguyen QD, Jaffe GJ, Slakter JS, Simader C, Soo Y, Schmelter T, Yancopoulos GD, Stahl N, Vitti R, Berliner AJ, Zeitz O, Metzig C, Korobelnik JF: Intravitreal aflibercept for diabetic macular edema: 100week results from the VISTA and VIVID studies. Ophthalmology 2015;122:2044-2052.

5 Do DV, Nguyen QD, Boyer D, Schmidt-Erfurth U, Brown DM, Vitti R, Berliner AJ, Gao B, Zeitz O, Ruckert R, Schmelter T, Sandbrink R, Heier IS; da Vinci Study Group: One-year outcomes of the da Vinci Study of VEGF Trap-Eye in eyes with diabetic macular edema. Ophthalmology 2012;119:1658-1665.

-6 Brown DM, Nguyen QD, Marcus DM, Boyer DS, Patel S, Feiner L, Schlottmann PG, Rundle AC, Zhang J, Rubio RG, Adamis AP, Ehrlich JS, Hopkins JJ; RISE and RIDE Research Group: Long-term outcomes of ranibizumab therapy for diabetic macular edema: the 36-month results from two phase III trials: RISE and RIDE. Ophthalmology 2013;120: 2013-2022.

-7 Wykoff CC, Elman MJ, Regillo CD, Ding B, Lu N, Stoilov I: Predictors of diabetic macular edema treatment frequency with ranibizum$\mathrm{ab}$ during the open-label extension of the RIDE and RISE trials. Ophthalmology 2016; 123:1716-1721.

8 Antonetti DA, Barber AJ, Bronson SK, Freeman WM, Gardner TW, Jefferson LS, Kester M, Kimball SR, Krady JK, LaNoue KF, Norbury CC, Quinn PG, Sandirasegarane L, Simpson IA; JDRF Diabetic Retinopathy Center Group: Diabetic retinopathy: seeing beyond glucose-induced microvascular disease. Diabetes 2006;55:2401-2411.

9 Funatsu H, Noma H, Mimura T, Eguchi S, Hori S: Association of vitreous inflammatory factors with diabetic macular edema. Ophthalmology 2009;116:73-79.

10 Zhang W, Liu H, Rojas M, Caldwell RW, Caldwell RB: Anti-inflammatory therapy for diabetic retinopathy. Immunotherapy 2011;3: 609-628.
11 Tang J, Kern TS: Inflammation in diabetic retinopathy. Prog Retin Eye Res 2011;30:343358.

12 Kim JH, Lee TG, Lew YJ: Short-term efficacy of intravitreal triamcinolone acetonide for bevacizumab-resistant diabetic macular oedema. Acta Ophthalmol 2015;93:e178-e179.

13 Chang-Lin JE, Attar M, Acheampong AA, Robinson MR, Whitcup SM, Kuppermann BD, Welty D: Pharmacokinetics and pharmacodynamics of a sustained-release dexamethasone intravitreal implant. Invest Ophthalmol Vis Sci 2011;52:80-86.

14 Boyer DS, Faber D, Gupta S, Patel SS, Tabandeh H, Li XY, Liu CC, Lou J, Whitcup SM; Ozurdex CHAMPLAIN Study Group: Dexamethasone intravitreal implant for treatment of diabetic macular edema in vitrectomized patients. Retina 2011;31:915-923.

15 Boyer DS, Yoon YH, Belfort R Jr, Bandello F, Maturi RK, Augustin AJ, Li XY, Cui H, Hashad Y, Whitcup SM; Ozurdex MEAD Study Group: Three-year, randomized, sham-controlled trial of dexamethasone intravitreal implant in patients with diabetic macular edema. Ophthalmology 2014;121:1904-1914.

-16 Callanan DG, Gupta S, Boyer DS, Ciulla TA, Singer MA, Kuppermann BD, Liu CC, Li XY, Hollander DA, Schiffman RM, Whitcup SM; Ozurdex PLACID Study Group: Dexamethasone intravitreal implant in combination with laser photocoagulation for the treatment of diffuse diabetic macular edema. Ophthalmology 2013;120:1843-1851.

17 Dugel PU, Bandello F, Loewenstein A: Dexamethasone intravitreal implant in the treatment of diabetic macular edema. Clin Ophthalmol 2015;9:1321-1335.

18 Campochiaro PA, Hafiz G, Mir TA, Scott AW, Zimmer-Galler I, Shah SM, Wenick AS, Brady CJ, Han I, He L, Channa R, Poon D, Meyerle C, Aronow MB, Sodhi A, Handa JT, Kherani S, Han Y, Sophie R, Wang G, Qian J: Pro-permeability factors in diabetic macular edema; the diabetic macular edema treated with Ozurdex trial. Am J Ophthalmol 2016;168:13-23.

19 Totan Y, Guler E, Guragac FB: Dexamethasone intravitreal implant for chronic diabetic macular edema resistant to intravitreal bevacizumab treatment. Curr Eye Res 2016;41: 107-113.

20 Esen E, Sizmaz S, Demircan N: Efficacy of dexamethasone intravitreal implant for the treatment of persistent diffuse diabetic macular edema. Int Ophthalmol 2017;37:1-6.

21 Scaramuzzi M, Querques G, Spina CL, Lattanzio R, Bandello F: Repeated intravitreal dexamethasone implant (Ozurdex) for diabetic macular edema. Retina 2015;35:12161222.

22 Coscas G, Coscas F, Zucchiatti I, Glacet-Bernard A, Soubrane G, Souied E: SD-OCT pattern of retinal venous occlusion with cystoid macular edema treated with Ozurdex ${ }^{\circledR}$. Eur J Ophthalmol 2011;21:631-636.
23 Bansal P, Agarwal A, Gupta V, Singh R, Gupta A: Spectral domain optical coherence tomography changes following intravitreal dexamethasone implant, Ozurdex ${ }^{\circledR}$ in patients with uveitic cystoid macular edema. Indian J Ophthalmol 2015;63:416-422.

24 Otani T, Yamaguchi Y, Kishi S: Correlation between visual acuity and foveal microstructural changes in diabetic macular edema. Retina 2010;30:774-780.

25 Reznicek L, Cserhati S, Seidensticker F, Liegl R, Kampik A, Ulbig M, Neubauer AS, Kernt M: Functional and morphological changes in diabetic macular edema over the course of anti-vascular endothelial growth factor treatment. Acta Ophthalmol 2013;91:e529-e536.

26 Gillies MC, Lim LL, Campain A, Quin GJ, Salem W, Li J, Goodwin S, Aroney C, McAllister IL, Fraser-Bell S: A randomized clinical trial of intravitreal bevacizumab versus intravitreal dexamethasone for diabetic macular edema: the BEVORDEX study. Ophthalmology 2014;121:2473-2481.

27 Escobar-Barranco JJ, Pina-Marin B, Fernandez-Bonet M: Dexamethasone implants in patients with naive or refractory diffuse diabetic macular edema. Ophthalmologica 2015; 233:176-185.

28 Gutierrez-Benitez L, Millan E, Arias L, Garcia $\mathrm{P}$, Cobos E, Caminal M: Dexamethasone intravitreal implants for diabetic macular edema refractory to ranibizumab monotherapy or combination therapy. Arch Soc Esp Oftalmol 2015;90:475-480.

29 Querques G, Darvizeh F, Querques L, Capuano V, Bandello F, Souied EH: Assessment of the real-life usage of intravitreal dexamethasone implant in the treatment of chronic diabetic macular edema in France. J Ocul Pharmacol Ther 2016;32:383-389.

30 Maturi RK, Pollack A, Uy HS, Varano M, Gomes AM, Li XY, Cui H, Lou J, Hashad Y, Whitcup SM, Ozurdex MSG: Intraocular pressure in patients with diabetic macular edema treated with dexamethasone intravitreal implant in the 3-year MEAD study. Retina 2016;36:1143-1152.

31 Totan Y, Güler E, Güragac FB: Dexamethasone intravitreal implant for chronic diabetic macular edema resistant to intravitreal bevacizumab treatment. Curr Eye Res 2016;41: 107-113.

32 Bressler SB, Qin H, Beck RW, Chalam KV, Kim JE, Melia M, Wells JA 3rd; Diabetic Retinopathy Clinical Research Network: Factors associated with changes in visual acuity and central subfield thickness at 1 year after treatment for diabetic macular edema with ranibizumab. Arch Ophthalmol 2012;130:11531161.

33 NICE: Ranibizumab for treating diabetic macular oedema. February 2013. https:// www.nice.org.uk/guidance/ta274/chapter/1guidance.
DEX Implant in DME and SD-OCT Predictive Factors
Ophthalmologica DOI: $10.1159 / 000485852$ 
-34 Bandello F, Cunha-Vaz J, Chong NV, Lang GE, Massin P, Mitchell P, Porta M, Prunte C, Schlingemann R, Schmidt-Erfurth U: New approaches for the treatment of diabetic macular oedema: recommendations by an expert panel. Eye (Lond) 2012;26:485-493.

-35 Sun JK, Lin MM, Lammer J, Prager S, Sarangi R, Silva PS, Aiello LP: Disorganization of the retinal inner layers as a predictor of visual acuity in eyes with center-involved diabetic macular edema. JAMA Ophthalmol 2014; 132:1309-1316.
36 Bolz M, Schmidt-Erfurth U, Deak G, Mylonas G, Kriechbaum K, Scholda C; Diabetic Retinopathy Research Group Vienna: Optical coherence tomographic hyperreflective foci: a morphologic sign of lipid extravasation in diabetic macular edema. Ophthalmology 2009; 116:914-920.

37 Chatziralli IP, Sergentanis TN, Sivaprasad S: Hyperreflective foci as an independent visual outcome predictor in macular edema due to retinal vascular diseases treated with intravitreal dexamethasone or ranibizumab. Retina 2016;36:2319-2328.
38 Hasegawa N, Nozaki M, Takase N, Yoshida M, Ogura Y: New insights into microaneurysms in the deep capillary plexus detected by optical coherence tomography angiography in diabetic macular edema. Invest Ophthalmol Vis Sci 2016;57:OCT348-OCT355.

-39 Lee J, Moon BG, Cho AR, Yoon YH: Optical coherence tomography angiography of DME and its association with anti-VEGF treatment response. Ophthalmology 2016;123:23682375. 